\title{
ASSESSING DER FLEXIBILITY IN A GERMAN DISTRIBUTION NETWORK FOR DIFFERENT SCENARIOS AND DEGREES OF CONTROLLABILITY
}

\author{
André SILVA \\ INESC TEC - Portugal \\ andre.c.silva@inesctec.pt \\ Leonel CARVALHO \\ INESC TEC - Portugal \\ leonel.m.carvalho@inesctec.pt \\ Ricardo BESSA \\ INESC TEC - Portugal \\ ricardo.j.bessa@inesctec.pt
}

\author{
Jean SUMAILI \\ INESC TEC - Portugal \\ jean.sumaili@inesctec.pt \\ Luís SECA \\ INESC TEC - Portugal \\ luis.seca@inesctec.pt \\ Gunnar SCHAARSCHMIDT \\ RWE - Germany \\ Gunnar.Schaarschmidt@rwe.com
}

\author{
João SILVA \\ INESC TEC and FEUP - Portugal \\ joao.v.silva@inesctec.pt \\ Manuel MATOS \\ INESC TEC and FEUP - Portugal \\ mmatos@inesctec.pt \\ Roland HERMES \\ RWE - Germany \\ Roland.Hermes@rwe.com
}

\begin{abstract}
This paper evaluates the flexibility provided by distributed energy resources (DER) in a real electricity distribution network in Germany. Using the Interval Constrained Power Flow (ICPF) tool, the maximum range of flexibility available at the primary substation was obtained for different operation scenarios. Three test cases were simulated, differing mainly in the considered level of renewable energy sources (RES) production. For each test case, the obtained results enabled the construction of flexibility areas that define, for a given operating point, the limits of feasible values for the active and reactive power that can be exchanged between the TSO and the DSO. Furthermore, the tool can also be used to evaluate the contribution from each type of DER to the overall distribution network flexibility.
\end{abstract}

\section{INTRODUCTION}

Within the Smart Grids (SG) paradigm, the future electricity distribution system will be characterized by more observability and controllability, offering the conditions for bidirectional control of Distributed Energy Resources (DER) with high penetration of Distributed Renewable Energy Resources (DRES). The flexibilities available in the network may be used to solve local technical problems such as under/overvoltage or branch congestion. They may be also used in order to meet regulatory restrictions and to avoid penalties or to offer flexibilities to the TSO.

Today, in Germany, the scheduled operating point at the primary substation must fit in the TSO rules. Therefore, the DSO optimizes the scheduled operating point to avoid penalties and to find the minimum cost by using an optimal power flow (OPF). In the future, it is also in discussion whether the DSO should provide the TSO with a specific operating point as an additional service. The Interval Constrained Power Flow (ICPF) tool was/has been developed in the framework of FP7 EU Project evolvDSO (www.evolvdso.eu) [1] and is able to provide the maximum range of flexibility available at the primary substation and how it varies according to the cost that the DSO is willing to pay for activating it without violating technical constraints. As such, the ICPF tool works in the TSO-DSO coordination, with the main goal of estimating the flexibility range [2] at the TSO-DSO boundary, by aggregating the distribution network flexibility in order to enable a technical and economic evaluation of the flexibility, from the bulk power system point of view.

In this paper, simulations conduced for a real distribution network in Germany are presented and analyzed. The estimated region of feasible values of active and reactive power are referred to a specific connection with the transmission system, which stands as the distribution system boundary node. Although it can be possible within the ICPF tool, none of the simulations considered any restrictive cost, since the main focus is centered on finding the aggregated flexibility boundaries of the distribution network for each simulated test case.

\section{FLEXIBILITY ASSESSMENT}

The simulated German distribution network is a meshed HV network with connected HV/MV transformers. These power transformers are equipped with tap changers to control the MV voltage level. Despite having multiple connections to the transmission system, only one of them was considered for simulation purposes. The remaining connections were considered as having fixed power exchanges. For confidentiality reasons, all the power values presented in this paper have been rescaled using the same constant in order to keep the ratio between active and reactive power.

Table 1 - Network simulation cases main features

\begin{tabular}{|c|c|c|c|c|}
\hline \multicolumn{2}{|c|}{ Snapshot } & Case A & Case B & Case C \\
\hline \multicolumn{2}{|c|}{ RES Production Level } & $66 \%$ & $93 \%$ & $9 \%$ \\
\hline \multirow{2}{*}{$\begin{array}{l}\text { Wind Park } \\
\text { Generation }\end{array}$} & MW & 0.186 & 0.404 & 0.003 \\
\hline & Mvar & 0.015 & 0.023 & 0.002 \\
\hline \multicolumn{2}{|c|}{ Biomass Generation (MW) } & \multicolumn{3}{|c|}{0.243} \\
\hline \multirow{2}{*}{$\begin{array}{c}\text { Total Net } \\
\text { Load }\end{array}$} & MW & 1.114 & 1.092 & 1.555 \\
\hline & Mvar & 0.779 & 0.555 & 0.580 \\
\hline
\end{tabular}

Table 1 provides a brief description of the three snapshots that were used as base study test cases for the simulation scenarios construction. Each snapshot represents a distinct operating point for the analyzed distribution network. As it can be seen, these cases differ mostly in terms of the considered RES production level. The 
simulation scenarios were built upon the presented cases, considering distinct flexibility sources. Such sources include transformers tap changers, RES active and reactive power control and also storage devices. Due to the large number of existing transformers and also the meshed nature of the network, it can be expected that a considerable amount of reactive power flexibility will appear by means of tap changing transformers. As the MV grid is not modelled in this first approach, the complete flexibility of the tap changers is available for the optimization. In the real grid environment additional restrictions coming from MV voltage management will necessarily reduce this flexibility. The reference values for wind and biomass generation can be observed in Table 2, represented by their generation capacity limits considering two distinct time horizons: (i) as it is currently and (ii) as it is expected to be installed in the upcoming years. Biomass power plants were considered to be at its nominal power for all test cases. When considered as flexibility sources, such power plants can reduce their active power injection until its technical minimum, at about $40 \%$ of their rated nominal power. Wind parks and all the distributed wind units were considered to be at $66 \%, 93 \%$ and $9 \%$ load factor for cases A, B and C, respectively. All the RES generation can be curtailed.

Table 2 - Considered RES types and main characteristics

\begin{tabular}{|c|c|c|c|c|}
\hline Type & $\begin{array}{c}\mathrm{P}_{\min , \text { current }} \\
(\mathrm{MW})\end{array}$ & $\begin{array}{c}\mathrm{P}_{\max , \text { current }} \\
(\mathrm{MW})\end{array}$ & $\begin{array}{c}\mathrm{P}_{\min , \text { future }} \\
(\mathrm{MW})\end{array}$ & $\begin{array}{c}\mathrm{P}_{\max , \text { future }} \\
(\mathrm{MW})\end{array}$ \\
\hline Biomass & 0.097 & 0.243 & 0.117 & 0.290 \\
\hline Wind Park & 0 & 0.469 & 0 & 1.129 \\
\hline Distributed Wind & 0 & 0.179 & 0 & 0.431 \\
\hline
\end{tabular}

As for reactive power control (Q(U) control), a fixed power factor for all wind and biomass generation units was defined. For the storage devices two integration options were considered: (i) centralized storage at two primary substations, each device with $\pm 0.056 \mathrm{MW}$, and (ii) same amount of storage, but distributed over all the HV/MV substations. For all the conduced simulations, no demand flexibility was considered as the results for storage can be transferred to demand response as well. However, as the future distribution networks will offer more observability and controllability, the possibility of using demand management to increase the network flexibility represents an interesting thought.

\section{SIMULATION TEST CASES DESCRIPTION}

Based on each distribution network snapshot a simulation test case was created. For cases A, B and C, six scenarios were constructed. For test case A an extra scenario was defined, only with transformer tap changers, in order to assess the provided flexibility. Tables 3 and 4 describe the constructed test scenarios.

In the next section, the obtained results for the different wind production and load levels will be described.
Table 3 -Simulation specifications for test case A

\begin{tabular}{|c|c|c|c|}
\hline \multirow{2}{*}{ Scenario } & \multirow{2}{*}{ Description } & \multicolumn{2}{|c|}{ Flexibility Range } \\
\hline & & MW & Mvar \\
\hline 0 & Only taps & --- & --- \\
\hline status quo & taps + redispatch (existing) & 0.146 & --- \\
\hline 2 & $\begin{array}{l}\text { status quo + RES curtail and } \\
\mathrm{Q}(\mathrm{U}) \text { control (only new*) }\end{array}$ & 0.269 & 0.118 \\
\hline 3 & $\begin{array}{l}\text { status quo + RES curtail and } \\
\mathrm{Q}(\mathrm{U}) \text { control (existing + new*) }\end{array}$ & 0.381 & 0.301 \\
\hline $4 \& 5$ & $\begin{array}{c}2+\text { storage devices (central } \\
\text { and distributed) }\end{array}$ & 0.491 & 0.118 \\
\hline 6 & superposition of all the above & 0.825 & 0.301 \\
\hline
\end{tabular}

*new installed capacity is the difference between $\mathrm{P}_{\text {future }}$ and $\mathrm{P}_{\text {current }}$

Table 4 - Simulation specifications for test cases B and $C$

\begin{tabular}{|c|c|c|c|c|c|}
\hline \multirow{2}{*}{ Scenario } & \multirow{2}{*}{ Description } & \multicolumn{4}{|c|}{ Flexibility Range } \\
\cline { 3 - 6 } & & \multicolumn{2}{|c|}{ Case B } & \multicolumn{2}{|c|}{ Case C } \\
\cline { 3 - 6 } & MW & Mvar & MW & Mvar \\
\hline quo & $\begin{array}{c}\text { taps + Q(U) } \\
\text { control for current } \\
\text { RES capacity }\end{array}$ & --- & 0.577 & --- & 0.188 \\
\hline 1 & $\begin{array}{c}\text { status quo }+ \\
\text { redispatch }\end{array}$ & 0.146 & 0.577 & 0.146 & 0.188 \\
\hline 2 & $\begin{array}{c}1+\text { wind curtail } \\
\text { (only new*) }\end{array}$ & 1.038 & 0.577 & 0.206 & 0.188 \\
\hline 3 & $\begin{array}{c}2+\text { wind curtail } \\
\text { (existing + new*) }\end{array}$ & 1.672 & 0.577 & 0.249 & 0.188 \\
\hline 4 & $\begin{array}{c}3+\text { centralized } \\
\text { storage devices }\end{array}$ & 1.894 & 0.577 & 0.470 & 0.188 \\
\hline 5 & $\begin{array}{c}3+\text { distributed } \\
\text { storage devices }\end{array}$ & 1.894 & 0.577 & 0.470 & 0.188 \\
\hline
\end{tabular}

\section{SIMULATION RESULTS}

In this section, the results of the developed simulations for each test case will be presented and thoroughly analyzed. For each test case, the results are compared with the ones obtained for previous scenarios. With this approach, it will be possible to show the evolution of the results throughout the scenarios.

\section{Moderate RES production - Case A}

Test case $\mathrm{A}$ is based upon a snapshot of the German distribution network characterized by a moderate level of RES production (wind generation is at $66 \%$ load factor). The obtained results are presented in Figure 1 and Table 5. It can be seen that from scenario 0 to scenario 6 the obtained flexibility ranges for both active and reactive power grows. This result is in accordance with the defined criteria from Table 3.

For scenario 0 , only transformer tap changers variations were allowed. As it was expected, by only acting over the transformers a significant range of reactive power flexibility ( $0.356 \mathrm{Mvar}$ ) becomes available. This result is, like stated before, related with the fact that a significant number of transformers with tap changing capability is connected in the network, providing support for voltage control. The calculated range is a theoretical maximum, neglecting MV voltage control which is the primary objective for the tap changers. As for active power, no significant flexibility range is obtained, since no 
additional active power generation/absorption was considered. The observed small variation can be attributed mainly to power losses (of about $0.027 \mathrm{MW}$ ).

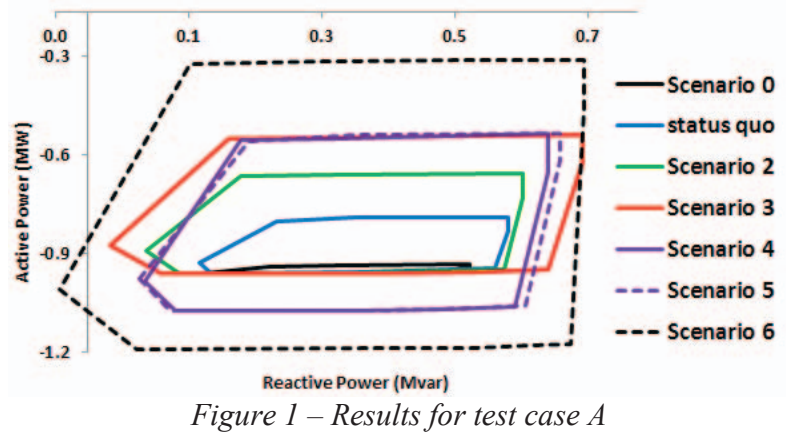

Scenario 1 allows only biomass curtailment for the current installed capacity. Thus, the verified active power flexibility range is increased. As only curtailment is allowed, its limits are only increased upwards.

Table 5 - Results for test case A

\begin{tabular}{|c|c|c|}
\hline \multirow{2}{*}{ Scenario } & \multicolumn{2}{|c|}{ Obtained Flexibility Range } \\
\cline { 2 - 3 } & MW & Mvar \\
\hline 0 & 0.027 & 0.356 \\
\hline status quo & 0.166 & 0.412 \\
\hline 2 & 0.295 & 0.503 \\
\hline 3 & 0.409 & 0.631 \\
\hline $4 \& 5$ & 0.508 & 0.584 \\
\hline 6 & 0.850 & 0.701 \\
\hline
\end{tabular}

Scenarios 2 and 3 depart from scenario 1, but allow for RES active power curtailment and reactive power control. For scenario 2 only the forcoming wind generation capacity is considered. As for scenario 3, the total wind generation capacity matches the one planned for the future. It can be observed that both active and reactive power flexibility ranges are widened, since more flexibility was allowed.

Scenarios 4 and 5 take into account the possibility of using storage devices as sources of flexibility, resulting in an expected active power flexibility range increase. As it can be observed in Figure 1, since the storage allows bidirectional power exchange, both the upwards and downwards flexibility ranges have in fact increased. When comparing the obtained results for both scenarios, no significant difference between central and distributed storages is observed. This may be explained by the fact that the network does not reach its technical limits (voltage limits and branch capacities) within the considered scenario. Scenario 6 flexibility area covers all its previous scenarios areas. Such a result is in accordance with the expected results, since it considers the superposition of the defined scenarios for the future.

High and Low RES production - Cases B and C Test case $\mathrm{B}$ considers wind generation at $93 \%$ of the installed capacity. This is the highest of all the simulation cases. As so, it could be expected that case B would have the larger flexibility area when compared to the remaining cases. This can be confirmed by looking at
Figure 2 and at the summary Table 6.

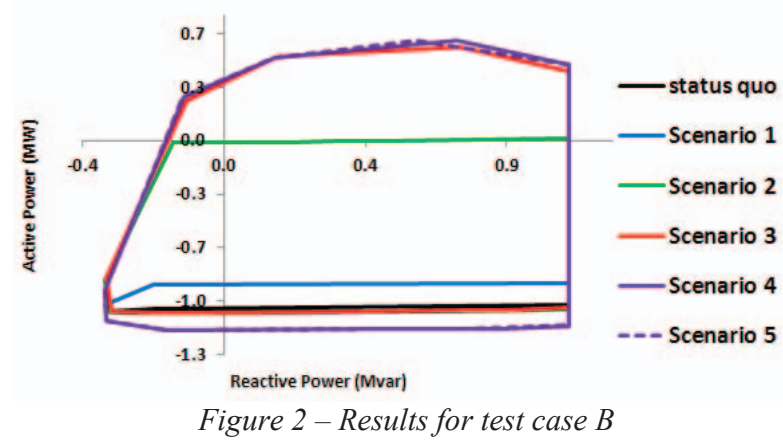

The status quo scenario allows only for transformer tap changers variations and existing RES reactive power control. The obtained results reveal a significant reactive power flexibility range (1.456 Mvar) and only a slight active power flexibility range $(0.046 \mathrm{MW})$.

From scenarios 1 to 5 , no additional reactive power flexibility was considered, so the obtained ranges stay nearly the same. Active power flexibility varies for such scenarios, so the obtained areas also vary. As the allowed active power curtailment grows between scenarios, the obtained range also increases. As previously observed for test case $\mathrm{A}$, the addition of storage devices allows not only the increase in the upward flexibility active power limits, but also for the downwards limits, which corresponds to active power injection.

Table 6-Results for test cases B and C

\begin{tabular}{|c|c|c|c|c|}
\hline \multirow{2}{*}{ Scenario } & \multicolumn{3}{|c|}{ Obtained Flexibility Range } \\
\cline { 2 - 5 } & \multicolumn{2}{|c|}{ Case B } & \multicolumn{2}{c|}{ Case C } \\
\cline { 2 - 5 } & MW & Mvar & MW & Mvar \\
\hline status quo & 0.046 & 1.456 & 0.024 & 0.656 \\
\hline 1 & 0.181 & 1.459 & 0.177 & 0.699 \\
\hline 2 & 1.085 & 1.461 & 0.236 & 0.712 \\
\hline 3 & 1.654 & 1.456 & 0.282 & 0.720 \\
\hline 4 & 1.802 & 1.460 & 0.502 & 0.758 \\
\hline 5 & 1.804 & 1.458 & 0.500 & 0.756 \\
\hline
\end{tabular}

An interesting outcome of test case B, can be noticed by observing the resultant flexibility areas for scenarios 3, 4 and 5. By comparing Table 6 with Table 4 , it can be seen that for such scenarios wider active power ranges would be expected. For example, the defined criteria for scenario 5 accounted for a total of $1.894 \mathrm{MW}$ of allowed flexibility, but the obtained range was only of $1.804 \mathrm{MW}$. This effect indicates that the network maximum operating point has been reached, given the allowed flexibility criteria. This means that even if more flexibility was added, it would never be used, since it would certainly lead to the violation of the network branch flow limits. A more thorough inspection of the network branch flows revealed that for those scenarios, some transmission lines close to the considered boundary node are operating at their technical limits.

Test case $\mathrm{C}$ is based on a snapshot for which the studied network is operating with a low level of RES production (at $9 \%$ of the installed capacity). The available flexibility sources and scenario criteria follow the same rules as 
previously defined for test case $\mathrm{B}$. The obtained results were expected to be similar in nature to those of case B but, since less wind generation was available, the obtained flexibility areas should be smaller. Figure 3 and Table 6 present the obtained results.

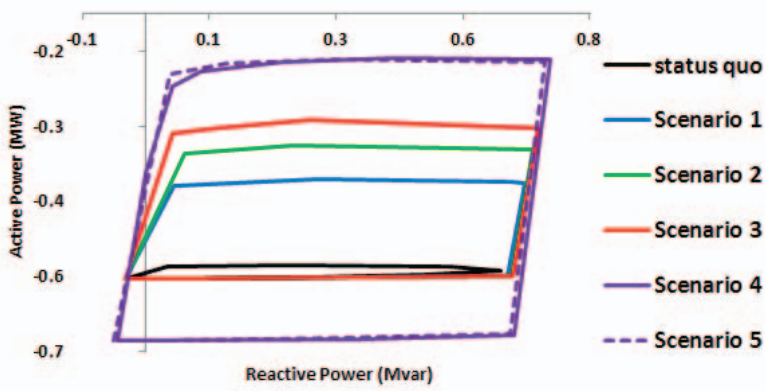

Figure 3 - Results for test case C

Status quo scenario revalidates the significance of the voltage regulation performed by transformer tap changers, particularly for highly inductive networks such as the HV/MV ones. The obtained reactive power flexibility range equals 0.656 Mvar, while the range for active power amounts only to $0.024 \mathrm{MW}$ (power losses). For the remaining scenarios, it is possible to notice that the obtained flexibility area increases from scenarios 1 to 5. This occurs only for case C, since the system's operating point is far from its technical limits and no active power flexibility is wasted as proven by Table 6 results. Like previously observed for case $\mathrm{B}$, there is no significant difference between the obtained results for the central and distributed storage scenarios.

\section{Comparison with Monte Carlo Sampling (MCS)}

The performance of the ICPF tool can be evaluated through two operational Key Performance Indicators (KPIs) originally defined for evolvDSO project. The flexibility area obtained with the ICPF tool can be compared with the flexibility area achieved through MCS. Therefore, the flexibility area increase can be measured. The computational time reduction results from the comparison between the time of the power flows that were run in the MCS and the time necessary for ICPF to define the flexibility area.

Table 7 -Operational KPIs for test case B

\begin{tabular}{|c|c|c|c|c|c|c|}
\hline \multirow{3}{*}{ Scenario } & \multicolumn{3}{|c|}{ Area increase (\%) } & \multicolumn{3}{c|}{ Time reduction (\%) } \\
\cline { 2 - 7 } & $\begin{array}{c}1000 \\
\text { samples }\end{array}$ & $\begin{array}{c}10000 \\
\text { samples }\end{array}$ & $\begin{array}{c}100000 \\
\text { samples }\end{array}$ & $\begin{array}{c}1000 \\
\text { samples }\end{array}$ & $\begin{array}{c}10000 \\
\text { samples }\end{array}$ & $\begin{array}{c}100000 \\
\text { samples }\end{array}$ \\
\hline status quo & 93.9 & 91.4 & 87.3 & 74.4 & 97.7 & 99.8 \\
\hline 1 & 95.8 & 93.7 & 91.2 & 75.9 & 97.7 & 99.8 \\
\hline 2 & 94.1 & 92.2 & 90.0 & 54.0 & 95.3 & 99.6 \\
\hline 3 & 92.9 & 89.9 & 86.7 & 58.5 & 95.6 & 99.6 \\
\hline 4 & 92.1 & 90.1 & 86.4 & 69.3 & 96.9 & 99.7 \\
\hline 5 & 94.8 & 89.9 & 87.0 & 58.0 & 95.8 & 99.6 \\
\hline
\end{tabular}

The MCS was run for 1000, 10000 and 100000 randomly extracted samples. To avoid repetition, only case B is presented. The analysis of the two operational KPIs in Table 7 for the studied network allowed to verify that the ICPF tool provides a better description of the network operating limits when compared to the Monte Carlo Simulation results. The ICPF tool allows for the identification of rare events, increasing the size of the estimated flexibility area, since the MCS based on sampling had some difficulties in finding feasible points close to the boundary of the flexibility area. ICPF also provides a considerable reduction on the computational effort.

\section{CONCLUSIONS}

For every case regarded in this exemplary analysis, the obtained flexibility areas were verified as being coherent with the pre-simulation flexibility criteria, except for Case B scenarios 3, 4 and 5, where it was observed that the network limits were reached. Thus, any additional flexibility would not contribute for the flexibility area increase. This confirms that a meshed topology network has a significant impact regarding its operational flexibility, due to the amount of transformers with tap changers. In this analysis as a first approach the theoretical impact of the transformers is analyzed, neglecting voltage control needs in the underlying MV network. In further steps it would be necessary to evaluate the realistic flexibilities by integration the full model of the MV network in the simulation.

The obtained results analysis proved that even based on today's DSO flexibilities it is possible to offer a broad range of different operating points. Main flexibility is provided by tap changers and grid-tie inverter flexibilities of DRES units as required in the German grid code. The use of storage has also shown to increase the flexibility range. However, there is no significant difference between the ranges obtained for the centralized and distributed storage. In the near future, the German distribution networks will be able to provide even more flexibility due the increasing penetration of RES with high controllability of reactive power and possible wind curtailment respecting the limitations given by the infrastructure.

\section{ACKNOWLEDGMENTS}

The research leading to this work is being carried out as a part of the evolvDSO project (Development of methodologies and tools for new and evolving DSO roles for efficient DRES integration in distribution networks) www.evolvdso.eu. This project is funded by the European Commission under the seventh framework program (FP7) under grant agreement number 608732.

\section{REFERENCES}

[1] J. Sumaili et al., "Advanced methodologies and tools for operation and maintenance of distribution grids with DRES," Deliverable D3.3, FP7 EU Project evolvDSO, Sept. 2015.

[2] M. Heleno, R. Soares, J. Sumaili, R. Bessa, L. Seca, M. Matos, "Estimation of the flexibility range in the transmission-distribution boundary," in Proc. the IEEE PowerTech 2015, Eindhoven, NL, June 2015. 